\title{
Exploring Chemical Kinetics at Home in Times of Pandemic: Following the Bleaching of Food Dye Allura Red Using a Smartphone
}

\author{
Lorean Madriz,* Franco M. Cabrerizo, and Ronald Vargas
}

Cite This: https://doi.org/10.1021/acs.jchemed.0c01427

Read Online

ABSTRACT: In this communication, a remote experimental activity in chemical kinetics is described, taking into account the quantification based on the optical sensor of a smartphone. The objective pursued herein is to equip students with the appropriate tools and strategies required to empirically determine the parameters of the rate law including reaction orders, rate constant $(k)$, frequency factor $(A)$, and activation energy $\left(E_{\mathrm{a}}\right)$. Typical results of the proposed protocol are shown and discussed in the framework of the bleaching reaction of food dye allura red ( $\mathrm{RD} 40)$ and hypochlorite, as a representative example. A graphical approach of the concentration vs time data measured under the experimental condition where $[\mathrm{RD} 40] \ll\left[\mathrm{ClO}^{-}\right]$ (isolation method) suggests a first-order kinetics with respect to the dye. In addition, the analysis of the pseudo-first-order constant $\left(k_{\text {obs }}\right)$ shows a firstorder relationship with respect to $\mathrm{ClO}^{-}$. In addition, using the two-point form

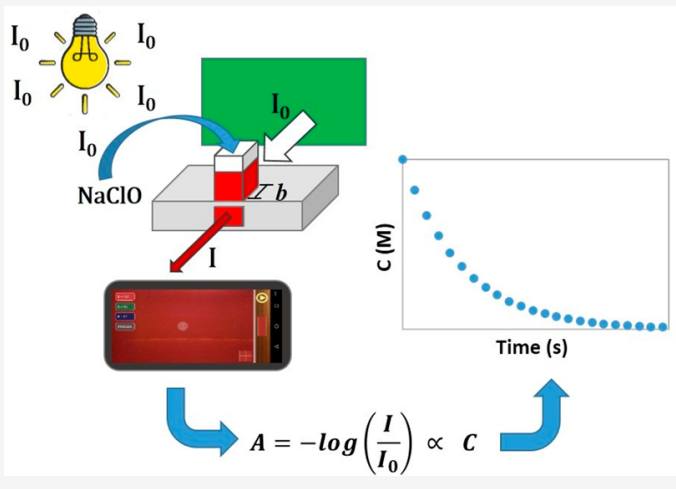
of the Arrhenius equation, values of $3.22 \times 10^{7} \mathrm{~s} / \mathrm{M}$ and $44.55 \mathrm{~kJ} / \mathrm{mol}$ were obtained for $A$ and $E_{a}$, respectively. Interestingly, all the kinetic parameters (reaction orders, $k, A$, and $E_{\mathrm{a}}$ ) are on the same order of magnitude as those previously reported in the literature and acquired with more sophisticated and accurate equipment. This experience provides evidence that it is possible to proceed with remote experimental activities to deepen the collection and analysis of kinetic data during a pandemic.

KEYWORDS: Hands-On Learning/Manipulatives, Second-Year Undergraduate, Physical Chemistry, Kinetics, Rate Law, $U V-V i s$ Spectroscopy

\section{INTRODUCTION}

Due to the recent COVID-19 pandemic, a rather big challenge has been the change from a face-to-face practical course to an online course, specifically in the area of experimental sciences. To this end, valid protocols were developed for conducting hands-on qualitative experiments at home, employing many available kitchen, cleaning, cosmetic, pharmacy, and garden chemicals that can be used to illustrate those principles shown in "traditional" chemistry courses at the various levels of education. ${ }^{1}$ However, at this point, a valid question would be the following: How far can we take these protocols in terms of quantitative analysis?

Recently, it has been reported that accessible protocols implement smartphones as key elements in the spectroscopic quantification of colored substances, especially as a tool for taking measurements at home. ${ }^{2,3}$ When light passes through a colored substance, the intensity of the photons coming out of the vessel or cuvette will be lower, due to the fact that molecules with double bonds and heteroatoms absorb a fraction of the incident light. Now, using the optical sensor of a smartphone, it is possible to measure photons and perform spectrophotometric measurements. ${ }^{2,3}$ This protocol only requires natural light, the smartphone as a photodetector, and a colored substance, making it very convenient to teach spectroscopy and instrumentation concepts remotely.

To further correlate the measured quantity with the substance that absorbs the incident light (chromophore), one must know the energy that this substance can absorb. This generally depends on the structure of the molecule because the colored substances absorb a part of the visible radiation that comes from the electromagnetic spectrum (Figure 1A). Light can be measured on the basis of its composition in terms of the intensity of its primary colors: red, green, and blue (RGB). Using the color wheel shown in Figure 1B, the color of the solution to be studied is located (reflected color), and the intensity of the opposite or complementary color (absorbed

Received: November 27, 2020

Revised: April 8, 2021 
(A)

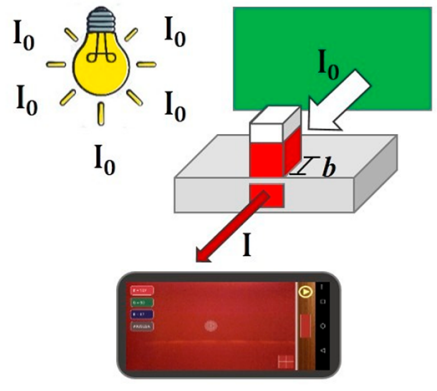

(B)

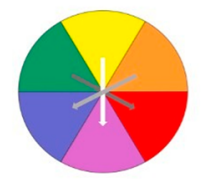

(C)

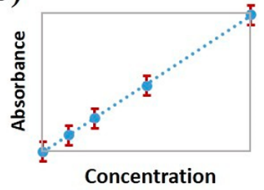

Figure 1. (A) Experimental scheme of the smartphone-based spectrophotometer for absorbance measurements. (B) Chromatic circle with complementary colors. (C) Graphic representation of the Lambert-Beer law.

color) is taken as the basis of measurement. Then, the intensity of light absorbed by the sample can be measured with an RGB analyzer such as those found in mobile phones. ${ }^{2}$ Finally, quantification can be made from the intensity measurement, according to eq 1 , where $A$ is the absorbance, $I_{0}$ is the intensity of the incident light, and $I$ is the intensity of the transmitted light. With this knowledge, through the Lambert-Beer law (eq 2) it will be possible to correlate the absorbance measured from a given sample with the concentration $(C)$ of the substance of interest (Figure 1C). Thus, the constant of proportionality is the product of the molar extinction coefficient $(\varepsilon)$ with the optical path of the reaction cell $(b)$.

$$
A=-\log \left(\frac{I}{I_{0}}\right)
$$

$$
A=\varepsilon b C
$$

Then, if a colored substance is available, its concentration can be measured, and this protocol can be implemented to follow the progress of a reaction where the consumption of reagent or the appearance of products leads to a significant change in the color intensity of the sample solution. A good example, of special interest, is the bleaching reaction, which normally involves redox processes. One of the oxidizing agents used in bleaching is hypochlorite ion, which attacks mainly by adding double bonds and aromatic rings and can also promote hydrogen abstraction. ${ }^{4}$

The bleaching reaction in azo-type dyes in neutral or basic media is given by the nucleophilic attack of $\mathrm{ClO}^{-}$on $-\mathrm{N}=$ $\mathrm{N}-$, leading to the asymmetric cleavage of the dye, and since this reaction occurs with peracids, $\mathrm{Cl}^{-}$is expelled as an outgoing group. ${ }^{4}$ Said reaction can be carried out on a substance such as allura red or red dye 40 (RD40), found in the worldwide commercial beverage by Coca-Cola Company: PowerAde Fruit Punch flavored at a concentration of $94 \mu \mathrm{M}^{5}$ This dye has an azo group attached to aromatic rings whose $\pi$ system is the responsible of the chromophoric properties of the dye (Figure 2). Note that this dye is widely used as an additive in food, beverage, cosmetic, and pharmaceutical products. ${ }^{5}$

The objective of this work is to provide a quantitative experimental design to allow students to learn, at home, chemical kinetics concepts, making use of the benefits of smartphone technology and widely available chemicals and materials. In particular, smartphones are used as a homemade simple beam spectrophotometer to study the bleaching reaction of $\mathrm{RD} 40$ by sodium hypochlorite (an oxidizing

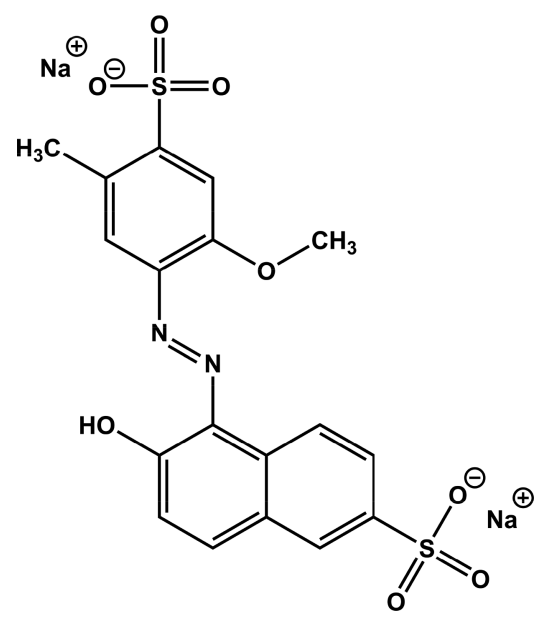

Figure 2. Structure of RD40 or disodium 6-hydroxy-5-((2-methoxy-5methyl-4-sulfophenyl)azo)-2-naphthalenesulfonate.

agent present in several commercial cleaning products, i.e., $\mathrm{NaOCl} 2.67 \%$ aqueous solution). In addition, second-year science and engineering students will be able to familiarize themselves with the generation and analysis of kinetic data from home.

\section{EXPERIMENTAL SECTION}

\section{Materials}

This experiment requires the following materials:

- PowerAde Fruit Punch flavored commercial beverage (Coca-Cola Company: 94.0 $\mu \mathrm{M}$ RD40)

- Héroe commercial bleach water solution (Fadial, S.R.L.:: $25 \mathrm{~g} \mathrm{Cl} / \mathrm{L}$, equivalent to $0.70 \mathrm{M} \mathrm{ClO}^{-}$)

- Disposable syringe or measuring container

- Cardboard box as cell holder that allows the passage of light only through the optical path

- Green card stock as a background to reflect light

- Transparent and smooth container as sample cell (e.g., lipstick cap)

- Reusable and freezable plastic ice cubes

- Stopwatch

- Visible light lamp or flashlight

- Smartphone

Students must download an application, either "ColorMeter Free" for Android or "Colorometer" for iPhone. In this work, a G6 smartphone from Motorola with an Android operative system was used.

\section{Experimental Setup}

The smartphone-based spectrophotometer was configured as reported in the literature. ${ }^{2}$ Briefly, a cardboard box was used in which the corresponding holes were made to place: (i) a transparent container as a reaction cell from the top and (ii) a smartphone as a light receiver from the front. The green card was placed behind the sample box, which was arranged so that the sample could be seen through and a space left between them so that light could enter (Figure 1A). The smartphone was placed with the ColorMeter Free application open; this was placed so that the camera lens is parallel to the hole where the container with the study solution is located. To avoid fluctuations in measurements, the system remained fixed once assembled. It is recommended to provide constant illumination through a visible light lamp or flashlight. An example of a setup 
made by the students who carried out the experiments described at home, is shown in the Supporting Information.

\section{Calibration}

Using a disposable syringe, serial dilutions of the commercial beverage were made in half each time $(47.0,32.5,11.8$, and 5.9 $\mu \mathrm{M})$. Tap water was taken as a blank in order to record the reference $\mathrm{G}$ component $\left(I_{0}\right)$ measurements on the smartphone screen. $I_{\mathrm{G}}$ measurements were converted to absorbance according to eq 1 , and the calibration curve for the dye was performed. Measurements were made in triplicate.

\section{Kinetic Experiments: Variation of the Initial Concentration} and Temperature

The bleaching reaction was tested by varying the concentration of dye and hypochlorite, maintaining a constant volume equal to $5 \mathrm{~mL}$. An aliquot of PowerAde (solution A) was placed in the cell reactor, and the initial measurement of $I_{\mathrm{G}}$ was recorded; then, an aliquot of hypochlorite solution prepared with a commercial bleach $(\mathrm{mL})$ :tap water $(\mathrm{mL})$ ratio as solution B (280 mM, 2:3) or solution C (140 mM, 1:4) was added, and the recording of $G$ began with the start of the stopwatch. The measurement was performed every $10 \mathrm{~s}$ until a time of $60 \mathrm{~s}$ passed, and then every $20 \mathrm{~s}$ until a final time of $100 \mathrm{~s}$ was reached. To record the intensity value, it is possible to use the application's print screen option and copy the G magnitude. For each experiment, the temperatures were measured with a thermometer at the beginning and at the end of the reaction. The experiments described above were carried out at room temperature $\left(18{ }^{\circ} \mathrm{C}\right)$. In addition, one set of experiments was carried out at $4{ }^{\circ} \mathrm{C}$ by surrounding the cell with reusable and prefreezable plastic ice cubes. Table 1 indicates the mixed aliquots of the dye and hypochlorite solutions.

Table 1. Volumes of RD40 and Hypochlorite Solutions for Conducting the Experiments

\begin{tabular}{cccc} 
& \multicolumn{3}{c}{ Solutions, mL } \\
\cline { 2 - 4 } Experiment & $V_{\mathrm{A}}, 94 \mu \mathrm{M} \mathrm{RD} 40$ & $V_{\mathrm{B}}, 280 \mathrm{mM} \mathrm{ClO}$ & $V_{\mathrm{C}}, 140 \mathrm{mM} \mathrm{ClO}$ \\
1 & 4.0 & 0.0 & 1.0 \\
2 & 4.5 & 0.5 & 0.0 \\
3 & 4.5 & 0.0 & 0.5 \\
4 & 4.0 & 0.0 & 1.0
\end{tabular}

The procedure to determine the kinetic parameters from the proposed experiments requires verifying the linearity of a $\log ([\mathrm{RD} 40])$ vs time plot when $[\mathrm{RD} 40] \ll\left[\mathrm{ClO}^{-}\right]$, which will indicate that the order of reaction with respect to $\mathrm{RD} 40(\alpha)$ is equal to 1 . Then, for each experiment, the observed kinetic constant $\left(k_{\text {obs }}\right)$ can be estimated as: $\ln (10)$ multiplied by the negative of the slope that results from the best linear fit of the $\log ([\mathrm{RD} 40])$ vs time plots. Note that if $\ln ([\mathrm{RD} 40])$ vs time plot is used, $k_{\text {obs }}$ are obtained directly from the negative of the slope. Now, to calculate the reaction order with respect to hypochlorite $(\beta)$, the ratio of two values of the observed kinetic constant (e.g., $\left.\left(k_{\text {obs }}\right)_{2} /\left(k_{\text {obs }}\right)_{3}\right)$ was taken. The conditions that allow an easy calculus of the reaction order were those that had an equal value for $[\mathrm{RD} 40]_{0}$ but different values for $\left[\mathrm{ClO}^{-}\right]_{0}$; as $\left(k_{\text {obs }}\right)_{2} /\left(k_{\text {obs }}\right)_{3}=\left\{\left[\mathrm{ClO}^{-}\right]_{2} /\left[\mathrm{ClO}^{-}\right]_{3}\right\}^{\beta}$, it is possible to solve the last equation to find the $\beta$ value. Finally, $E_{\mathrm{a}}$ and $A$ are estimated by solving a system of equations that result from substituting kinetic data at two different temperatures into the Arrhenius equation. The key considerations of this calculation procedure and other details are discussed in the Results and Discussion section.

\section{HAZARDS}

Commercial sodium hypochlorite should be handled with care. It is a respiratory irritant and harmful if swallowed. Do not let it touch your skin or your clothing. If anything is spilled, clean the area with plenty of water. All chemicals and solution may be disposed of down the drain.

\section{RESULTS AND DISCUSSION}

Figure $3 \mathrm{~A}$ shows the setup to measure the intensity of light through the sample used at home. Likewise, Figure $3 \mathrm{~B}$ shows
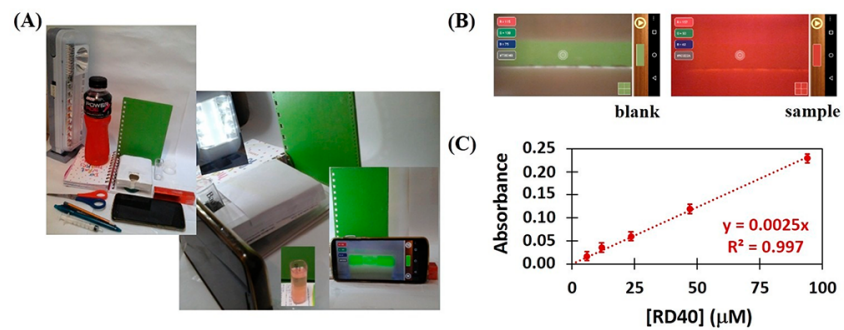

Figure 3. (A) Experimental setup of homemade smartphone-based spectrophotometer used for measurements. (B) Visualization of solutions blank (tap water) and RD40 through the screen of the smartphone. Notice the boxes with the intensities in RGB code in the upper-left corner. (C) Plot of absorbance of RD40 solutions vs concentration.

the smartphone screen pointing at the sample and indicating the RGB intensity values; the blank solution is tap water, and therefore, the green tone of the background cardboard color is observed. Upon placement of a solution of the dye $(5.0 \mathrm{~mL})$, the screen turns red. Then, the intensity value of the complementary color to the analyte absorption can be recorded, that is, the green component G. The absorbance can be calculated by entering in eq 1 the measured values of intensity $\mathrm{G}$ in both situations: blank and analyte solutions.

Figure $3 \mathrm{C}$ presents an absorbance vs concentration plot of the different dilutions of the original sample, observing a linear relationship consistent with the Lambert-Beer law (eq 2). Therefore, it was verified that the variations in the optical density of the solutions, due to the lower number of chromophore molecules in the dye solutions, are quantifiable using the optical sensor of the smartphone. The typical deviation between the three replicates was around 5\%, and the limit of detection ( 3 times the standard deviation of the blank solution) was $13 \mu \mathrm{M}$. This is especially useful for remote home experimentation, as the calibration curve obtained will check the concentration of dye in another aqueous solution of interest. $^{2,3}$ Precisely, what will be done is quantify the concentration of the dye after adding $\mathrm{ClO}^{-}$and measure it at different time intervals.

Now, to carry out an evaluation of the kinetics of a chemical process, the exploration of the effect of the concentration of the reagents should be considered, in this case, RD40 and $\mathrm{ClO}^{-}$, in addition to the temperature. This should be supported by experimental measurements, which quantify the variation in the concentration of at least one reagent or product. ${ }^{6}$ At this point, we will take advantage of the smartphone's optical sensor to follow the color change of the dye solution. Table 2 indicates the concentrations of 
Table 2. Experimental Conditions, Concentrations, and Pseudo-First-Order Kinetic Constants $\left(k_{\mathrm{obs}}\right)$

\begin{tabular}{|c|c|c|c|c|c|}
\hline Experiment & Temperature (K) & {$\left[\mathrm{ClO}^{-}\right](\mathrm{mM})$} & {$[\mathrm{RD} 40](\mu \mathrm{M})$} & $10^{3} \times k_{\mathrm{obs}}\left(\mathrm{s}^{-1}\right)$ & $R^{2}$ \\
\hline 1 & 291 & 28 & 75.2 & 9.08 & 0.956 \\
\hline 2 & 291 & 28 & 84.6 & 10.4 & 0.978 \\
\hline 3 & 291 & 14 & 84.6 & 5.75 & 0.953 \\
\hline 4 & 277 & 28 & 75.2 & 3.58 & 0.967 \\
\hline
\end{tabular}

(A)

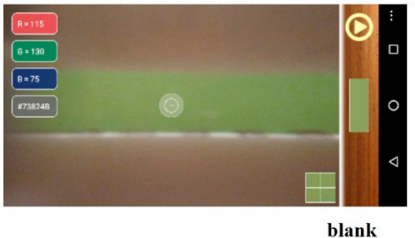

(B)

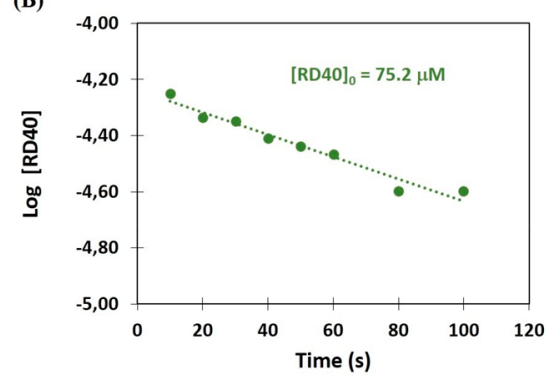

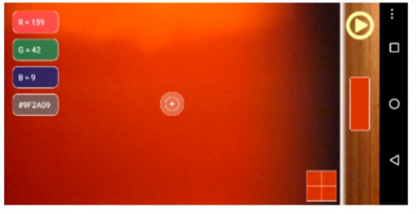

$\mathbf{t}=\mathbf{1 0} \mathrm{s}$

(C)

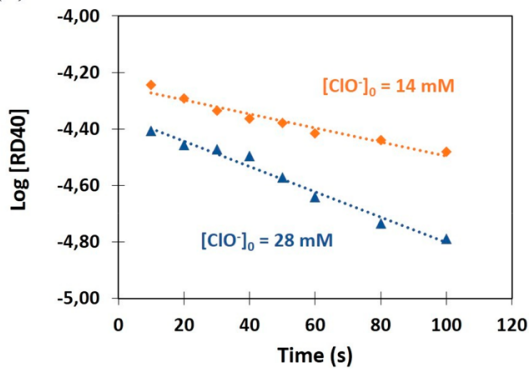

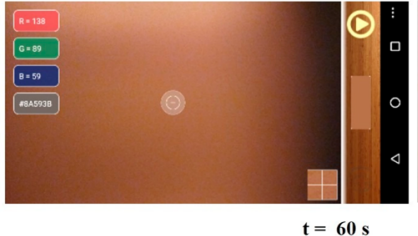

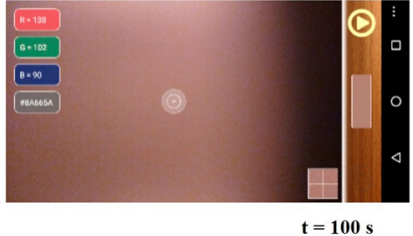

(D)

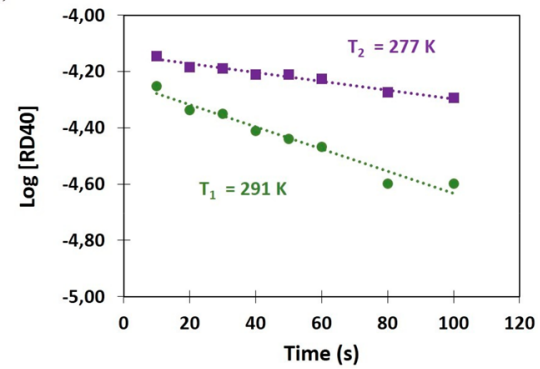

Figure 4. (A) View of blank and samples of RD40 during the bleaching reaction, and $\log ([\mathrm{RD} 40])$ vs time plots. $(\mathrm{B}) \mathrm{T}=291 \mathrm{~K},\left[\mathrm{ClO}{ }^{-}\right]_{0}=28$ $\mathrm{mM}$, and $[\mathrm{RD} 40]_{0}=75.2 \mu \mathrm{M}$. (C) $\mathrm{T}=291 \mathrm{~K},[\mathrm{RD} 40]_{0}=84.6 \mu \mathrm{M}$, and two different $\left[\mathrm{ClO}^{-}\right]_{0}$ values. (D) $[\mathrm{ClO}]_{0}=28 \mathrm{mM},[\mathrm{RD} 40]_{0}=75.2 \mu \mathrm{M}$, and two temperatures. Dotted lines are the first-order kinetic fits.

hypochlorite ion and RD40 when mixed according to the volumetric proportions indicated in the Experimental Section (Table 1). It should be noted that the concentration of RD40 is much lower than that of $\mathrm{ClO}^{-}$, so it is convenient to implement kinetic analysis based on the isolation method. ${ }^{6}$ Briefly, the rate $(r)$ of a bleaching reaction can be expressed as

$$
r=k[\mathrm{RD} 40]^{\alpha}\left[\mathrm{ClO}^{-}\right]^{\beta}
$$

where $\alpha$ and $\beta$ are the reaction orders, brackets indicate the reagents' concentrations, and $k$ is the rate constant. This depends on the temperature according to the Arrhenius equation:

$$
k=A \mathrm{e}^{-E_{\mathrm{a}} / R T}
$$

where $A$ is the frequency factor and $E_{\mathrm{a}}$ is the activation energy. The isolation method applied to the dye suggests that [RD40] $\ll\left[\mathrm{ClO}^{-}\right]$, and then

$$
r=k_{\mathrm{obs}}[\mathrm{RD} 40]^{\alpha}
$$

with $k_{\mathrm{obs}}$ being the observed reaction rate constant.

$$
k_{\mathrm{obs}}=k\left[\mathrm{ClO}^{-}\right]^{\beta}
$$

Figure $4 \mathrm{~A}$ shows the color change of the sample as a function of time, through the smartphone screen. Figure 4B,C shows the linearization of the experimental data, $\log ([\mathrm{RD} 40])$ vs time, highlighting that the variation in concentration responds to a pseudo-first-order kinetics and also depends on the initial amount of $\mathrm{ClO}^{-}$. Table 2 reports the $k_{\text {obs }}$ and $R^{2}$ values obtained in each experiment. From this analysis, it can be concluded that the order of reaction with respect to the dye is $\alpha=1$. Note that it is important to comment on two experimental aspects that must be addressed to minimize errors: (i) The timer must be started by adding the bleaching agent, and (ii) a sampling interval of $10 \mathrm{~s}$ is adequate considering the instrumental time response.

To determine the order of reaction with respect to $\mathrm{ClO}^{-}$, experiments were carried out whose initial concentration of dye was the same, but that of hypochlorite was different. Therefore, the ratio between the $k_{\mathrm{obs}}$ of experiments 2 and 3 indicated in Table 2 was calculated, and it was compared with the ratio between $k_{\text {obs }}$ deduced from eq 5 ; that is, $\left(k_{\text {obs }}\right)_{2} /$ $\left(k_{\text {obs }}\right)_{3}=\left\{\left[\mathrm{ClO}^{-}\right]_{2} /\left[\mathrm{ClO}^{-}\right]_{3}\right\}^{\beta}$. From the last equation, the reaction order can be calculated, obtaining $\beta=0.8$. This value is close to the reported reaction order, $\beta=0.95 \sim 1{ }^{7}$ and we will consider it appropriate, since the quantification using the smartphone and the experimentation with kitchen utensils at home are illustrative of the concepts.

Figure 4D helps us to visualize that the reaction rate decreases at a lower temperature, since it is noticeable that the resulting change in concentration is less under the same initial conditions of reagent concentration. To determine the parameters of the Arrhenius equation (eq 5), the values of $k$ were first calculated at the two temperatures studied (experiments 1 and 4$), k=k_{\mathrm{obs}} /\left[\mathrm{ClO}^{-}\right]$, resulting in $k\left(T_{1}=\right.$ $291 \mathrm{~K})=0.324 \mathrm{~s} / \mathrm{M}$ and $k\left(T_{2}=277 \mathrm{~K}\right)=0.128 \mathrm{~s} / \mathrm{M}$. So, the system of equations that arises from applying the logarithm to eq 5 and substitutes the two pairs of values $\left(T_{1}, k_{1}\right)$ and $\left(T_{2}\right.$, $k_{2}$ ) (see eqs 7 and 8) was solved. Finally, the search values for $A$ and $E_{\mathrm{a}}$ are reported in Table 3 .

$$
\ln \left(k_{1}\right)=\ln (A)-\frac{E_{\mathrm{a}}}{R T_{1}}
$$




$$
\ln \left(k_{2}\right)=\ln (A)-\frac{E_{\mathrm{a}}}{R T_{2}}
$$

Table 3. Comparison of Kinetic Parameters

\begin{tabular}{lll}
$\begin{array}{c}\text { Kinetic } \\
\text { Parameters }\end{array}$ & \multicolumn{1}{c}{ This Work } & \multicolumn{1}{c}{ Literature Value $^{a}$} \\
$\alpha$ & 1 & 1 \\
$\beta$ & $0.8 \pm 0.1$ & $0.95 \pm 0.07$ \\
$A(\mathrm{~s} / \mathrm{M})$ & $3.22 \times 10^{7} \pm 6.47 \times 10^{6}$ & $6.82 \times 10^{7} \pm 6.21 \times 10^{6}$ \\
$E_{\mathrm{a}}(\mathrm{kJ} / \mathrm{mol})$ & $44.55 \pm 6.01$ & $40.41 \pm 4.36$ \\
${ }^{a}$ See ref 7. & & \\
\hline
\end{tabular}

In general, the magnitudes of the kinetic parameters $(\alpha, \beta, k$, $A$, and $E_{\mathrm{a}}$ ) obtained for the RD40 bleaching reaction are in the order of magnitude reported. ${ }^{7}$ In the Supporting Information, there is results obtained by the students who carried out the experiments described from home, and discussion points to improve the meeting sessions between students and instructors. In any case, it will be indicated that the characteristic deviation of the kinetic parameters considered is $\pm 10-20 \%$. Furthermore, the resulting system is appropriate to be considered as a model in the remote teaching of experimental chemistry.

\section{CONCLUSIONS}

The proposed activity considers the use of the bleaching reaction to explore the reaction kinetic determination, making use of accessible and very low-cost instrumentation, achieving comparable results with experiments performed with high-tech equipment. It is worth mentioning that, despite the present work focusing on a particular chromophore, the protocol can be extended to other systems of interest. The foregoing will be decisive to maintain activities in the new technological era of distance education, currently catalyzed by the COVID-19 pandemic.

\section{ASSOCIATED CONTENT}

\section{Supporting Information}

The Supporting Information is available at https://pubs.acs.org/doi/10.1021/acs.jchemed.0c01427.

Information on the results obtained by the students who carried out the experiments described at home, and discussion points to improve the meeting sessions between students and instructors (PDF, DOCX)

\section{AUTHOR INFORMATION}

\section{Corresponding Author}

Lorean Madriz - Instituto de Investigaciones Fisicoquímicas Teóricas y Aplicadas (INIFTA), CCT-La Plata-CONICET, Universidad Nacional de La Plata (UNLP), 1900 La Plata, Argentina; (ㅇ) orcid.org/0000-0001-7476-7114;

Email: loreanmadriz@inifta.unlp.edu.ar

\section{Authors}

Franco M. Cabrerizo - Instituto Tecnológico de Chascomús (INTECH), Universidad Nacional de San Martín (UNSAM)-Consejo Nacional de Investigaciones Científicas y Técnicas (CONICET), B7130IWA Chascomús, Argentina; 으 orcid.org/0000-0001-9584-4369

\section{Ronald Vargas - Instituto Tecnológico de Chascomús (INTECH), Universidad Nacional de San Martín (UNSAM)-Consejo Nacional de Investigaciones Científicas y Técnicas (CONICET), B7130IWA Chascomús, Argentina; 다잉.org/0000-0002-4890-8187}

Complete contact information is available at:

https://pubs.acs.org/10.1021/acs.jchemed.0c01427

Notes

The authors declare no competing financial interest.

\section{ACKNOWLEDGMENTS}

We thank Rosa Erra-Balsells from Universidad de Buenos Aires, students from INTECH-UNSAM and TWAS-TYAN for the collaboration. F.M.C. and R.V. are members of the TWAS Young Affiliates Network (TYAN).

\section{REFERENCES}

(1) Selco, J. I. Using Hands-On Chemistry Experiments While Teaching Online. J. Chem. Educ. 2020, 97 (9), 2617-2623.

(2) Kuntzleman, T. S.; Jacobson, E. C. Teaching Beer's Law and Absorption Spectrophotometry with a Smart Phone: A Substantially Simplified Protocol. J. Chem. Educ. 2016, 93 (7), 1249-1252.

(3) Destino, J. F.; Cunningham, K. At-Home Colorimetric and Absorbance-Based Analyses: An Opportunity for Inquiry-Based, Laboratory-Style Learning. J. Chem. Educ. 2020, 97 (9), 2960-2966.

(4) Oakes, J.; Gratton, P. Kinetic Investigations of the Oxidation of Arylazonaphthol Dyes in Hypochlorite Solutions as a Function of $\mathrm{pH}$. J. Chem. Soc., Perkin Trans. 2 1998, 2, 2201-2206.

(5) Stevens, L. J.; Burgess, J. R.; Stochelski, M. A.; Kuczek, T. Amounts of Artificial Food Colors in Commonly Consumed Beverages and Potential Behavioral Implications for Consumption in Children: Revisited. Clin. Pediatr. 2014, 53 (2), 133-140.

(6) Levine, I. N. Physical Chemistry, 6th ed.; Mc Graw Hill: New York, 2009; pp 515-589.

(7) Ganley, J. C. A Homogeneous Chemical Reactor Analysis and Design Laboratory: The Reaction Kinetics of Dye and Bleach. Educ. Chem. Eng. 2015, 12, 20-26. 


\title{
Supporting Information
}

\section{Exploring chemical kinetic at home in times of pandemic: following the bleaching of food dye allura red using a smartphone}

\author{
Lorean Madriz, ${ }^{1, *}$ Franco M. Cabrerizo, ${ }^{2}$ and Ronald Vargas. ${ }^{2}$ \\ ${ }^{1}$ Instituto de Investigaciones Fisicoquímicas Teóricas y Aplicadas (INIFTA), CCT-La Plata-CONICET, \\ Universidad Nacional de La Plata (UNLP), Diag. 113 y 64, La Plata, Argentina. \\ 2Instituto Tecnológico de Chascomús (INTECH), Universidad Nacional de San Martín (UNSAM) - \\ Consejo Nacional de Investigaciones Científicas y Técnicas (CONICET), Av. Intendente Marino, Km 8.2, \\ CC 164 (B7130IWA), Chascomús, Argentina. \\ *E-mail: loreanmadriz@inifta.unlp.edu.ar
}

Information on the results obtained by two (2) students who performed the experiments described at home is presented below: Student $1(\mathbf{A}, \mathbf{C}, \mathbf{E}$ and $\mathbf{G})$ and Student 2 (B, D, F and H). Figure S1 (A) and (B) shows the setups to measure the intensity of light through the samples studied, $(\mathbf{C})$ and (D) shows the smartphone screen pointing at the blank solution (tap water) indicating the RGB intensity values, $(\mathbf{E})$ and $(\mathbf{F})$ shows the calibration curve of RD40 using Eqs.1 and 2 from the manuscript, and finally, $(\mathbf{G})$ and $(\mathbf{H})$ shows the concentration decay at two different reaction temperatures $\left(\mathbf{T}_{\mathbf{1}}=284.5 \mathrm{~K}\right.$ and $\left.\mathbf{T}_{\mathbf{2}}=277 \mathrm{~K}\right)$. Finally, $\boldsymbol{k}_{\mathrm{obs}}$ and $\boldsymbol{k}$ at the two temperatures studied and parameters of the Arrhenius equation ( $A$ and $E_{a}$. from Eq. 5) are reported in Table S1. It should be noted that the activation energy determined by the students $\left(E_{a}(1)=48.88 \mathrm{KJ} / \mathrm{mol}\right.$ and $\left.E_{a}(2)=35.42 \mathrm{KJ} / \mathrm{mol}\right)$ are in agreement with that reported in the manuscript $\left(E_{a}=38.82 \mathrm{KJ} / \mathrm{mol}\right)$. 
(A)

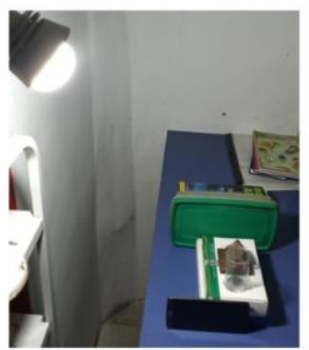

(B)

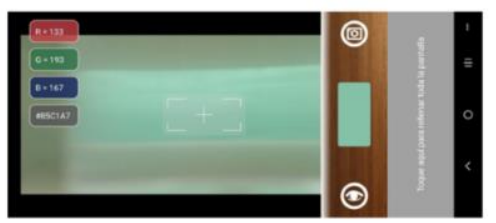

(C)

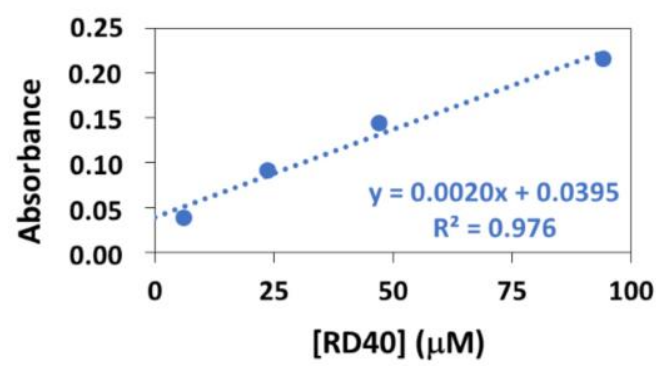

(D)

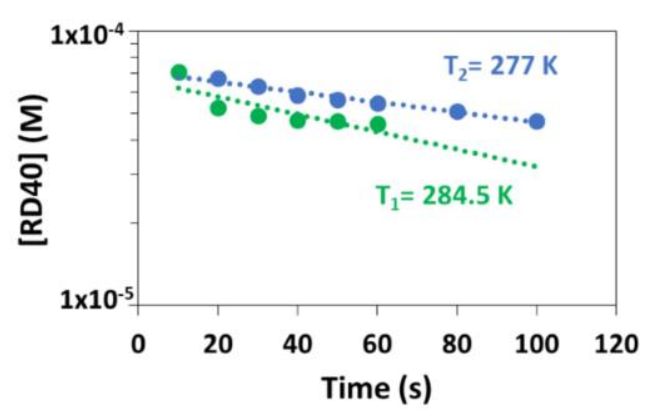

(E)

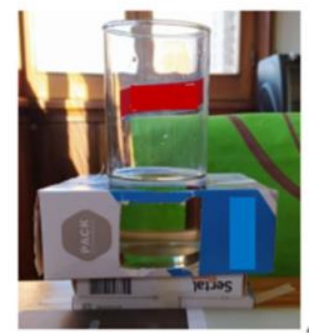

(F)

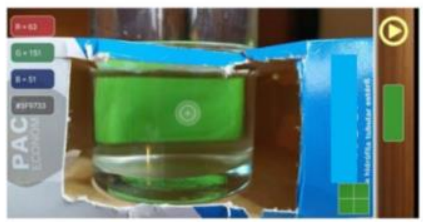

(G)

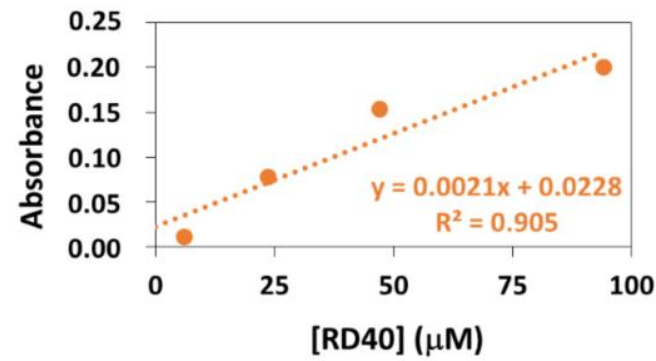

(H)

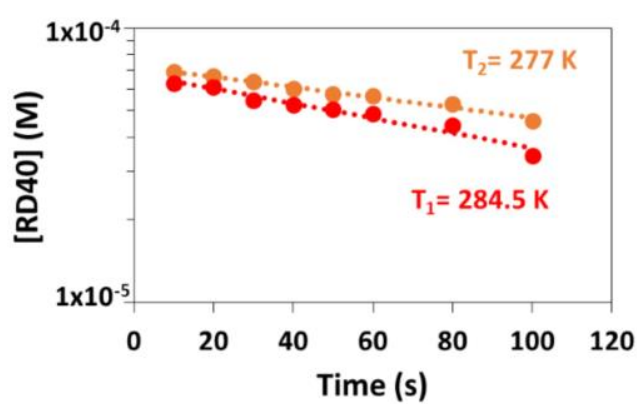

Figure S1. (A) and (B) Experimental setup of homemade smartphone-based spectrophotometer used for measurements, (C) and (D) visualization of solution blank (tap water) through the screen of the smartphone, (E) and (F) plot of absorbance of RD40 solutions vs. concentration, and (G) and (H) [RD40] vs. time semi logarithmic plots. Dotted lines are the first order kinetic fits. Student 1 (A, C, E and G) and Student 2 (B, D, F and H).

Table S1. Calculated parameters.

\begin{tabular}{ccc} 
Parameters & Student 1 & Student 2 \\
$k_{\text {obs }}\left(\mathrm{T}_{1}\right)\left(\mathrm{s}^{-1}\right)$ & 0.007 & 0.006 \\
$k_{\mathrm{obs}}\left(\mathrm{T}_{2}\right)\left(\mathrm{s}^{-1}\right)$ & 0.004 & 0.004 \\
$k\left(\mathrm{~T}_{1}\right)(\mathrm{s} / \mathrm{M})$ & 0.232 & 0.250 \\
$k\left(\mathrm{~T}_{2}\right)(\mathrm{s} / \mathrm{M})$ & 0.143 & 0.143 \\
$A(\mathrm{~s} / \mathrm{M})$ & $2.37 \times 10^{8}$ & $6.83 \times 10^{5}$ \\
$E_{\mathrm{a}}(\mathrm{KJ} / \mathrm{mol})$ & 48.88 & 35.42 \\
\hline
\end{tabular}


Some discussion ideas are pointed out to illustrate the possible improvements that could be implemented, and precisely those that must be clear to the student, to understand the value of carrying out experimentation in laboratories with the appropriate instrumentation.

a.- If the smartphone's optical sensor performs the measurement considering a range of wavelengths, then: how does the measurement affect when having: $(i)$ chromophores that absorb at very different intervals? and (ii) chromophores that adsorb in the same spectral region?.

b.- How is the previous point resolved if the measurements are made with a UV-Vis spectrophotometer?.

c.- Comment on the light losses and scattering in your experimental setup.

d.- By using volumetric instruments and a spectrophotometer with appreciations lower than $0.1 \%$, could the characteristic errors of the determined parameters be improved?.

e.- Could you carry out an experiment similar to the one proposed using the smartphone to measure the fluorescence of an organic molecule?. ${ }^{1}$

f.- Can the protocol be adapted to other dyes and their mixtures? ${ }^{2,3}$

g.- How does the ionic strength affect the performance of the reaction?, ${ }^{4}$ how can a high dissolved ion condition be set from home?

h.- After studying the mechanism of action of bleaching agents, what other agent could be used to achieve the objective of this lab?. ${ }^{5}$

\section{REFERENCES}

1. Levanov, A.V.; Isaikina, O. Ya. Determination of a Kinetic Law of Phosphorescence Decay Using a Conventional Photo Camera and Free Image Processing Software. J. Chem. Educ. 2020, 97 (9), 2685-2690.

2. Arce, J.; Betancourt, R.; Rivera, Y.; Pijem, J. The Reaction of a Food Colorant with Sodium Hypoclorite. A Student - Designed Kinetic Experiment. J. Chem. Educ. 1998, 75(9), 1142-1144.

3. Henary, M.M.; Russell, A.A. An Inexpensive Kinetic Study: The Reaction of of FD\&C Red \#3 (Erythrosin B) with Hypoclorite. J. Chem. Educ. 2007, 84(3), 480.

4. Kalmatsky, B. An Improvement to: The Reaction of FD\&C Red \#3 (Erythrosin B) with Hypoclorite. J. Chem. Educ. 2013, 90(12), 1708-1709.

5. Farr, J.P.; Smith, W.L.; Steichen, D. S. "Bleaching Agents". In Kirk-Othmer Encyclopedia of Chemical Technology. Vol 4. John Wiley \& Sons, Inc., 2003; pp 43-81. 